\title{
Dynamical Jahn-Teller Effect and Berry Phase in Positively Charged Fullerene I. Basic Considerations
}

\author{
Paolo De Los Rios, ${ }^{1,2 *}$, Nicola Manini, ${ }^{1,2 \dagger}$ and Erio Tosatti ${ }^{1,2,3 \ddagger}$ \\ ${ }^{1}$ Istituto Nazionale di Fisica della Materia (INFM) \\ ${ }^{2}$ International School for Advanced Studies (SISSA), Via Beirut 4, I-34013 Trieste, Italy \\ ${ }^{3}$ International Centre for Theoretical Physics (ICTP), P.O. Box 586, I-34014 Trieste, Italy
}

(September 11, 2018)

\begin{abstract}
We study the Jahn-Teller effect of positive fullerene ions ${ }^{2} \mathrm{C}_{60}^{+}$and ${ }^{1} \mathrm{C}_{60}^{2+}$. The aim is to discover if this case, in analogy with the negative ion, possesses a Berry phase or not, and what are the consequences on dynamical Jahn-Teller quantization. Working in the linear and spherical approximation, we find no Berry phase in ${ }^{1} \mathrm{C}_{60}^{2+}$, and presence/absence of Berry phase for coupling of one $L=2$ hole to an $L=4 / L=2$ vibration. We study in particular the special equal-coupling case $\left(g_{2}=g_{4}\right)$, which is reduced to the motion of a particle on a 5-dimensional sphere. In the icosahedral molecule, the final outcome assesses the presence/absence of a Berry phase of $\pi$ for the $h_{u}$ hole coupled to $G_{g} / H_{h}$ vibrations. Some qualitative consequences on ground-state symmetry,
\end{abstract}

\footnotetext{
*Email: delos@hp720.cm.sissa.it

${ }^{\dagger}$ Email: manini@sissa.it

${ }^{\ddagger}$ Email: tosatti@sissa.it
} 
low-lying excitations, and electron emission from $\mathrm{C}_{60}$ are spelled out.

Typeset using REVTEX 


\section{INTRODUCTION}

Non-adiabatic systems, where the quantum mechanics of electrons and that of ions are deeply entangled, are among the most fascinating in physics. In condensed matter, entire classes of phenomena such as superconductivity or charge-density waves are of this nature. Dynamical Jahn-Teller (DJT) centers (molecules and ions, impurities, nuclei) constitute perhaps the simplest "zero-dimensional" example of nonadiabatic behavior, and have attracted attention for over half a century [1].

An amusing anomaly has long been pointed out [2 [1] in certain simple DJT centers, where an $e$ electronic doublet is Jahn-Teller (JT) coupled to a vibrational ("pseudo-rotational") $E$ doublet. In modern language, this anomaly can be seen as a Berry phase [5,6]. In simple words, what happens in these systems is that there exists a class of closed paths (loops) in the $E$ coordinate space, regarded as classical, adiabatically transporting the electronic ground state from an initial value $\psi_{0}$ to a final value $e^{i \gamma} \psi_{0}=-\psi_{0}$, instead of into $\psi_{0}$ itself. There is a Berry phase $\gamma=\pi$, whose origin lies in the fact that the point of electronic degeneracy (the "conical point" [7, 3]) is irreducibly surrounded by the trajectory in vibron space.

This anomaly which is present at the adiabatic level, where the ionic coordinated are classical, has very interesting consequences when the full joint ion-electron problem is finally quantized. In the $e \otimes E$ case cited above, there appears a new conserved "pseudo-angular momentum" quantum number, whose values are half-integer [1],6]. Consequently, all vibronic levels become twofold degenerate, as appropriate to a rotor coupled to a pseudospin $\frac{1}{2}$ [1,6].

It is an interesting question therefore, to inquire whether other nontrivial cases of DJT are realized in nature, which also possess a Berry phase. High-symmetry polyatomic molecules are an obvious place to look at.

The fullerene molecule, $\mathrm{C}_{60}$, has a very high $\mathcal{I}_{h}$ symmetry, and a nondegenerate ground state. Because of high symmetry, however, fullerene ions, both negative and positive have a high orbital degeneracy, and are therefore good candidates. 
In our previous work, we have studied [8,9] the DJT problem of negatively-charged fullerene ions, where $1 \leq n \leq 5$ electrons partly fill the $t_{1 u}$ Lowest Unoccupied Molecular Orbital (LUMO) of the neutral molecule. This electronic state couples linearly to the fivefold degenerate $H_{g}$ vibrations. For odd electron number $n$, the spectrum of this coupled dynamical $t_{1 u} \otimes H_{g}$ system is affected by anomalies which can again be usefully described in terms of a Berry phase [5].

Among notable consequences, we have predicted the dominance of $p$-wave attachment for low-energy electrons [10], a negative pairing energy for odd $n$ [8,9], characteristic spectroscopic anomalies [11 and a mechanism for kinematic electron pairing [12 14]

From the mathematical point of view, nevertheless, the DJT of $\mathrm{C}_{60}^{-}$, namely $t \otimes H$, was not particularly new: the analytical machinery was basically the same as that previously studied by O'Brien [15, 16] for the $t \otimes(E \oplus T)$ equal coupling case.

In the present paper, we move on to analyze the problem of the JT effect in the positively charged fullerene ions $\mathrm{C}_{60}^{n+}$, inquiring in particular about the possible relevance of a Berry phase in these systems too. As it will turn out, the answer is again affirmative (though with some qualifications). Moreover, we shall deal in this case with the less trivial JT problems $h \otimes H$ and $h \otimes(G \oplus H)$, where the search for an adiabatic anomaly will now be done afresh.

Fullerene cations $\mathrm{C}_{60}^{n+}$ are obtained by removing $n \leq 10$ electrons from (i.e. adding $n \leq 10$ holes to) the fivefold degenerate $h_{u}$ Highest Occupied Molecular Orbital (HOMO) [17 19]. Our approach allows us to treat the one-hole case $(n=1)$ as well as the two-holes $(n=2)$ singlet case, in a way which is essentially analytical, albeit for a very idealized set of parameters.

A hole in the $\mathrm{HOMO}$ interacts with the $\mathrm{C}_{60}$ vibrational modes. We focus on modes which couple linearly with the hole density fluctuations (which in turn are of course quadratic in the hole wave function). Hence, the icosahedral symmetry of the molecule restricts the linearly coupled modes to those of the same symmetry as the irreducible representation of the group $\left(\mathcal{I}_{h}\right)$ contained in the symmetric product of a hole representation with itself [20]: 


$$
\left\{h_{u} \otimes h_{u}\right\}_{s} \approx a_{g} \oplus h_{g} \oplus\left(g_{g} \oplus h_{g}\right)
$$

The $\mathcal{I}_{h}$ group is a subgroup of the full rotation group $\mathrm{O}(3)$. Therefore, a general representation of $\mathrm{O}(3)$ decomposes into a finite number of representations of $\mathcal{I}_{h}$ [21]. In particular, the $L=2 \mathcal{D}^{(2 \pm)}$ representations correspond to $h_{g / u}$ in icosahedral symmetry, and $L=4$ $\mathcal{D}^{(4 \pm)}$ decomposes into $g_{g / u} \oplus h_{g / u}$. Equation (11) may be rewritten, in $\mathrm{O}(3)$ notation,

$$
\left\{\mathcal{D}^{(2-)} \otimes \mathcal{D}^{(2-)}\right\}_{s} \approx \mathcal{D}^{(0+)} \oplus \mathcal{D}^{(2+)} \oplus \mathcal{D}^{(4+)}
$$

In order to take advantage of spherical symmetry, and as a simplifying approximation at this initial stage, we neglect the icosahedral details of the molecular shape, and we study the hole-vibron coupling within the $\mathrm{O}(3)$ scheme, as though $\mathrm{C}_{60}$ was a perfect sphere [22]. We study here therefore the problem of $\mathcal{D}^{(2-)}$ holes coupled to $\mathcal{D}^{(0+)}, \mathcal{D}^{(2+)}$ and $\mathcal{D}^{(4+)}$ vibrations.

The coupling with a $\mathcal{D}^{(0+)}$ vibration is a trivial polaronic problem, solved exactly as a displaced oscillator, and is irrelevant here, since it shifts energies, but does not cause splittings. We concentrate on quadrupolar $\left(\mathcal{D}^{(2+)}\right)$ and hexadecapolar $\left(\mathcal{D}^{(4+)}\right)$ modes, which lead instead to a non-trivial JT effect. In order to simplify the notation, we shall switch from the $\mathrm{O}(3)$ to the $\mathrm{SO}(3)$ notation, omitting therefore the \pm signs for the inversion.

The Hamiltonian we consider has the following structure:

$$
H=H_{0}+H_{h-v}, H_{h-v}=H_{h-v}^{(2)}+H_{h-v}^{(4)},
$$

where $H_{0}$ describes a free (uncoupled) $\mathcal{D}^{(2)}$ hole and $\mathcal{D}^{(2)}, \mathcal{D}^{(4)}$ vibrations, and $H_{h-v}$ the hole-vibron interaction, namely

$$
\begin{aligned}
& H_{0}=\sum_{L=2,4} \hbar \omega_{L} \sum_{m=-L}^{L}\left(b_{L, m}^{\dagger} b_{L, m}+\frac{1}{2}\right)+(\epsilon-\mu) \sum_{\sigma=\uparrow, \downarrow} \sum_{m=-2}^{2} c_{m, \sigma}^{\dagger} c_{m, \sigma}, \\
& H_{h-v}^{(L)}=g_{L} \frac{\hbar \omega_{L}}{2} \sum_{\sigma, m_{1}, m_{2}}(-1)^{m_{2}}\left\langle L, m_{1} \mid 2,-m_{2} ; 2, m_{1}+m_{2}\right\rangle \times \\
& \times\left[b_{L, m_{1}}^{\dagger}+(-1)^{m_{1}} b_{L,-m_{1}}\right] c_{m_{2}, \sigma}^{\dagger} c_{m_{1}+m_{2}, \sigma} .
\end{aligned}
$$

Here, $\hbar \omega_{L}$ are the energies of the (harmonic) vibrations $b_{L, m}^{\dagger}, g_{L}$ are dimensionless coupling parameters, $<L, m \mid L_{1}, m_{1} ; L_{2}, m_{2}>$ are Clebsch-Gordan coefficients, and there is no loss 
of generality in choosing the energy zero so that $\epsilon=\mu$. For simplicity, we shall assume here a single $L=2$ and a single $L=4$ mode corresponding to $\mathcal{D}^{(2)}\left(=H_{g}\right)$ and $\mathcal{D}^{(4)}\left(=G_{g}+H_{g}\right)$ vibrons respectively. In real $\mathrm{C}_{60}$, the spectrum is more complex (see Tab. 回), involving 8 $H_{g}$ and $6 G_{g}$ modes. Of these, only two $H_{g}$ modes, namely $H_{g}(1)$ and $H_{g}(4)$ are actually derived from a $\mathcal{D}^{(2)}$ spherical vibration, while only two doublets, namely $G_{g}(1) \oplus H_{g}(2)$ and $G_{g}(4) \oplus H_{g}(6)$ are of $L=4$ origin [22]. The other modes derived from different angular momenta $(L=3,5,6,7)$ do not belong to a complete $L$-multiplet, and cannot be simply treated in spherical symmetry. Our work is therefore incomplete and of purely qualitative value. Nevertheless, we expect some of the features of the present simplified analysis to be of more general relevance. For example, close pairs of modes, such as $G_{g}(3) \oplus H_{g}(3)$, or $G_{g}(6) \oplus H_{g}(8)$, have no reason to behave qualitatively differently from our $\mathcal{D}^{(4)}$ mode, in spite of their different $L=6$ and $L=7$ origins. We will also find a "conservation of Berry phases": if a JT-coupled problem has a Berry phase, the extension of the vibron space with the addition of a mode that does not carry a Berry phase, leaves intact the original Berry phase. In our case, we will show that the Berry phase is coming exclusively from the $h_{u} \otimes G_{g}$ coupling, the addition of the $H_{g}$ modes changing the dynamical properties, but not the topological ones.

According to the different relative values of the four parameters $\left(\omega_{2}, g_{2}, \omega_{4}, g_{4}\right)$ in $H$, different regimes can be attained. In particular, when the coupling is extremely strong $\left(g_{L} \rightarrow \infty\right)$, the JT distortion is large, higher order terms (neglected in (4)) will in principle become relevant. In such a case, the final state tends to approach closely a static JT symmetry-broken distortion. When the coupling is instead large but finite, there is a semiclassical region, where quantum fluctuations are able to connect classical equivalent valleys via tunneling, and so restore the full symmetry while also generating characteristic low-energy tunneling excitations in the spectrum. The intermediate coupling region $g_{L} \approx 1$ is, as usual, the less predictable. However, the weak coupling limit $\left(g_{L} \rightarrow 0\right)$ finally simplifies again into a perturbative problem. If either $g_{4}$ or $g_{2}$ alternatively vanish, we will have the special cases of $\mathcal{D}^{(2)} \otimes \mathcal{D}^{(2)}$ or $\mathcal{D}^{(2)} \otimes \mathcal{D}^{(4)}$ JT respectively. A special case we shall consider 
in greatest detail below is that of equal coupling of degenerate oscillators $\omega_{2}=\omega_{4}, g_{2}=g_{4}$.

This paper is organized as follows. The weak-coupling region $g_{2}, g_{4}<<1$ is discussed in Sect. [1], obtaining approximate analytic expressions for the splittings and shifts in the spectrum. Next, we shall move to the opposite, strong-coupling case. Here, the classical JT minima of the Born-Oppenheimer potential energy surfaces are studied first (Sect. III); then, in Sect. IV, the ionic kinetic energy is introduced semiclassically to obtain a description of the low-energy quantum excitations in the strong-coupling limit. Exact numerical diagonalizations will be used to shed light on a few particular features of the intermediatecoupling regime. Finally, the relevance of these preliminary theoretical results to the physics of fullerene cations is discussed in Sect. $\nabla$.

\section{THE WEAK-COUPLING LIMIT: PERTURBATIVE APPROACH}

The weak-coupling limit in a JT problem is a coupled situation in which both the electronic and the vibronic degrees of freedom need to be treated on equal footing, as equally "fast". Their cooperative dynamics is strictly regulated by quantum mechanics, and no "classical degree of freedom" can be singled out in this limit, as opposed to the Berry-phase semiclassical approach. Nonetheless, the limit of small $g_{2}, g_{4}$ is easily treated by perturbation theory, and the results are useful in providing an exact match to the semiclassical spectrum on the opposite side of the range of coupling.

The JT coupling (4) splits and shifts the harmonic levels to second-order in $g_{L}$. We compute here these shifts for both the fivefold-degenerate ground state and the 70-fold degenerate one-vibron excitation in the 1-hole case and for equal frequencies $\hbar \omega_{2}=\hbar \omega_{4}=1$. As we have two small parameters, $g_{2}$ and $g_{4}$, and two different perturbing Hamiltonians,

$H_{h-v}^{(2)}$ and $H_{h-v}^{(4)}$, there is an infinite number of choices of the small perturbing parameter, depending for example on the ratio $g_{2} / g_{4}$. We compute the energy shifts for three special cases: $g_{4}=0$, i.e. perturbing with respect to $H_{h-v}^{(2)}, g_{2}=0$, using $H_{h-v}^{(4)}$ alone, and finally $g_{2}=g_{4}=g$, the equal coupling case. The coefficients for the three cases are collected in 
Tab. II], along with the appropriate $\mathrm{SO}(3)$ symmetry labels. The energy of a state (in units of $\hbar \omega)$ is thus given by

$$
\text { (number of vibrons })+(\text { coefficient in table }) \times g^{2}+\mathcal{O}\left(g^{4}\right) \text {, }
$$

where the number of vibrons is one for all the states except for the ground state at the first line.

We note on the second column five states, in the 1-vibron multiplet, whose shifts are $-\frac{1}{4} g_{2}^{2}$, i.e. the same as for the ground state. These states are readily identified as the 45 -fold degenerate state corresponding to the $\mathcal{D}^{(4)}$ vibron fundamental state (here uncoupled, since $\left.g_{4}=0\right)$. Parallel considerations apply to the uncoupled $\mathcal{D}^{(2)}$ vibron in the third column, recognizable by the shifts of $-\frac{9}{20} g_{4}^{2}$.

The equal-coupling case is interesting from the point of view of symmetry. Pooler studied the Hamiltonian (4) from an algebraic point of view [23], discovering that the equal frequencies - equal coupling case $\omega_{2}=\omega_{4}, g_{2}=g_{4}$, is actually characterized not only by the obvious $\mathrm{SO}(3)$ symmetry, but also by rotation symmetry in a 5-dimensional space ( $\mathrm{SO}(5)$ group). In the language of the $\mathrm{SO}(5)$ irreducible representations, characterized by two quantum numbers $[l, m]$ [24], the $\mathcal{D}^{(2)}$ hole is classified as a $[1,0]$ irreducible representation of $\operatorname{SO}(5)$, while the 14 -fold degenerate vibration $\left(\mathcal{D}^{(2)} \oplus \mathcal{D}^{(4)}\right)$ is a realization of the $[2,0]$ representation of $\mathrm{SO}(5)$ [25]. In the fourth column of Table 【, we note that the one-vibron level in the equal-coupling case splits into three levels with degeneracies 30,35 and 5 respectively. The levels of degeneracy 5 and 30 are readily seen as representations [1,0] and [3,0] of $\mathrm{SO}(5)$ [26]. The 35-fold degenerate level, by table 10-3 of reference [24] is identified with a $[1,2]$ representation, as indicated in the last column of Table [I]. As this large symmetry is an exact feature of the Hamiltonian, it is not limited to the perturbative regime, but will be carried along to all values of (equal-) coupling, and to the semiclassical limit (Sect. [V]) in particular.

To conclude, we note that, while the $L=0,1,5,6$ levels are exactly the same in the three perturbative regimes summarized in Tab. II], the $L=2,3,4$ levels (marked in Tab. 
[1] with symbols $\left.{ }^{\dagger},{ }^{\ddagger}, *\right)$, in the three cases, are instead different linear combinations of the same states: this fact has a very simple explanation. The $L=0,1$ levels uniquely come from the coupling of the $\mathcal{D}^{(2)}$ hole to the $\mathcal{D}^{(2)}$ vibron, and the $L=5,6$ levels derive from the coupling of the $\mathcal{D}^{(2)}$ hole to the $\mathcal{D}^{(4)}$ vibron. The $L=2,3,4$ levels can come from either of the previous couplings, and there is no a priori reason why they should not mix among themselves.

\section{TOPOLOGY OF THE JAHN-TELLER MANIFOLD AND THE BERRY PHASES}

In this section we want to understand if the $\mathrm{JT}$ hole-vibron coupling in $\mathrm{C}_{60}$ positive ions possesses a Berry phase or not. In order to do that, the canonical steps are the following: (i) find the manifold, in the space of classical ionic coordinates, formed by the set of equivalent minima of Hamiltonian (4) (the minima of the Born-Oppenheimer (BO) energy surface); (ii) study the adiabatic transport of the electronic ground state wave-function - in this case a unit vector spanning a five-dimensional sphere - while the ionic coordinates are taken along a closed path on their manifold. Representation (41) of Hamiltonian (33) is inconvenient for this purpose, and we first perform a transformation of the electronic and vibronic operators to a suitable representation.

\section{A. The real representation}

We start defining a vector of hole-destruction operators,

$$
C_{\sigma}=\left(c_{2 \sigma}, c_{1 \sigma}, c_{0 \sigma}, c_{-1 \sigma}, c_{-2 \sigma}\right)^{T} .
$$

In terms of this vector, the interaction Hamiltonian can be written as

$$
H_{h-v}^{(L)}=\sum_{\sigma} C_{\sigma}^{\dagger} \cdot B_{L} \cdot C_{\sigma}
$$


where $C_{\sigma}^{\dagger}$ indicates the adjoint transpose of (6), and $B_{2}$ and $B_{4}$ are $5 \times 5$ matrices whose elements are combinations of vibrational coordinates. Next, we apply the following transformation to the electronic operators

$$
\tilde{C}_{\sigma}=\left(\begin{array}{l}
\tilde{c}_{1 \sigma} \\
\tilde{c}_{2 \sigma} \\
\tilde{c}_{3 \sigma} \\
\tilde{c}_{4 \sigma} \\
\tilde{c}_{5 \sigma}
\end{array}\right)=\frac{1}{\sqrt{2}}\left(\begin{array}{ccccc}
i & 0 & 0 & 0 & i \\
1 & 0 & 0 & 0 & -1 \\
0 & 0 & \sqrt{2} i & 0 & 0 \\
0 & -i & 0 & i & 0 \\
0 & 1 & 0 & -1 & 0
\end{array}\right) C_{\sigma} .
$$

The transformed vector $\tilde{C}_{\sigma}$ contains the phase factors necessary to give a suitable symmetry to the transformed $B$-matrices.

The final step converts the vibron operators into their real coordinate representation:

$$
q_{L, m}=\sum_{\lambda=-L}^{L} M_{m, \lambda}\left(b_{L, \lambda}^{\dagger}+(-1)^{\lambda} b_{L,-\lambda}\right)
$$

where

$$
\begin{aligned}
M_{m, \lambda \neq 0} & =(2 \operatorname{sign}(m))^{-\frac{1}{2}}\left(\delta_{m, \lambda}+(-1)^{m} \operatorname{sign}(m) \delta_{m,-\lambda}\right), \\
M_{m, 0} & =\delta_{m, 0},
\end{aligned}
$$

and the masses of both oscillators are taken as unity.

We rewrite $H_{0}$ and $H_{h-v}$ after the final transformation as

$$
\begin{aligned}
H_{0} & =\sum_{L=2,4} \frac{\hbar \omega_{L}}{2} \sum_{l=-L}^{L}\left(-\partial_{L, l}^{2}+q_{L, l}^{2}\right) \\
H_{h-v}^{(L)} & =g_{L} \frac{\hbar \omega_{L}}{2} \sum_{\sigma} \tilde{C}_{\sigma}^{\dagger} \tilde{B}_{L} \tilde{C}_{\sigma},
\end{aligned}
$$

where the matrices $\tilde{B}_{2}$ and $\tilde{B}_{4}$, the transformed of $B_{2}$ and $B_{4}$ under (8), expressed in terms of $q_{L, m}$ (9) read 


$$
\tilde{B}_{2}=\frac{1}{\sqrt{7}}\left(\begin{array}{ccccc}
2 q_{2,0} & 0 & 2 q_{2,2} & \sqrt{3} q_{2,-1} & -\sqrt{3} q_{2,1} \\
0 & 2 q_{2,0} & 2 q_{2,-2} & -\sqrt{3} q_{2,1} & -\sqrt{3} q_{2,-1} \\
2 q_{2,2} & 2 q_{2,-2} & -2 q_{2,0} & -q_{2,-1} & -q_{2,1} \\
\sqrt{3} q_{2,-1} & -\sqrt{3} q_{2,1} & -q_{2,-1} & -q_{2,0}+\sqrt{3} q_{2,2} & -\sqrt{3} q_{2,-2} \\
-\sqrt{3} q_{2,1} & -\sqrt{3} q_{2,-1} & -q_{2,1} & -\sqrt{3} q_{2,-2} & -q_{2,0}-\sqrt{3} q_{2,2}
\end{array}\right),
$$

and

$$
\begin{aligned}
& \tilde{B}_{4}=\frac{1}{\sqrt{56}} \times \\
& \left(\begin{array}{ccccc}
\sqrt{\frac{2}{5}} q_{4,0}+\sqrt{14} q_{4,4} & \sqrt{14} q_{4,-4} & \sqrt{6} q_{4,2} & q_{4,-1}+\sqrt{7} q_{4,-3} & -q_{4,-1}+\sqrt{7} q_{4,3} \\
\sqrt{14} q_{4,-4} & \sqrt{\frac{2}{5}} q_{4,0}-\sqrt{14} q_{4,4} & \sqrt{6} q_{4,-2} & -q_{4,-1}-\sqrt{7} q_{4,3} & -q_{4,-1}+\sqrt{7} q_{4,-3} \\
\sqrt{6} q_{4,2} & \sqrt{6} q_{4,-2} & \sqrt{\frac{72}{5}} q_{4,0} & \sqrt{12} q_{4,-1} & \sqrt{12} q_{4,1} \\
q_{4,-1}+\sqrt{7} q_{4,-3} & -q_{4,1}-\sqrt{7} q_{4,3} & \sqrt{12} q_{4,-1} & -\sqrt{\frac{32}{5}} q_{4,0}-\sqrt{8} q_{4,2} & \sqrt{8} q_{4,-2} \\
-q_{4,1}+\sqrt{7} q_{4,3} & -q_{4,-1}+\sqrt{7} q_{4,-3} & \sqrt{12} q_{4,1} & \sqrt{8} q_{4,-2} & -\sqrt{\frac{32}{5}} q_{4,0}+\sqrt{8} q_{4,2}
\end{array}\right)
\end{aligned}
$$

\section{B. The Born-Oppenheimer energy minima}

The classical (purely static) solution of the JT problem consists in finding the minimum of the Born-Oppenheimer (BO) potential energy defined as the sum of the potential term in (11) plus the lowest eigenvalue of the hole-vibron interaction matrix

$$
\mathcal{H}\left(\vec{q}_{2}, \vec{q}_{4}\right)=g_{2} \frac{\hbar \omega_{2}}{2} \tilde{B}_{2}+g_{4} \frac{\hbar \omega_{4}}{2} \tilde{B}_{4}
$$

in the (5+9)-dimensional space of the classical $q_{L, m}$ coordinates. This classical minimum is generally not unique. More typically, there will be continuous sets of minima, forming well defined manifolds embedded in the multi-dimensional coordinates space [27]. We call one such set of minima of the adiabatic potential Jahn-Teller Manifold (JTM). The topology of this manifold crucially affects the possible presence of a Berry phase. We study the JTM

following the approach of Öpik and Pryce [4, more recently reformulated by Ceulemans in a clear and useful form [27]. 
Consider a generic one-fermion state

$$
|\eta\rangle=\sum_{j=1}^{5} \eta_{j} \tilde{c}_{j, \uparrow}^{\dagger}|0\rangle
$$

where without loss of generality, the $\eta_{j}$ can be taken real, with the normalization constraint

$$
\sum_{j=1}^{5} \eta_{j}^{2}=1
$$

The quantum amplitudes $\vec{\eta}$ live therefore on a $S_{4}$ sphere in 5-dimensions, which we indicate as the electronic sphere (ES).

Within this notation, it is convenient to rewrite the classical potential energy as

$$
V\left(\vec{q}_{2}, \vec{q}_{4}, \vec{\eta}\right)=\sum_{L=2,4} \frac{\hbar \omega_{L}}{2} \sum_{l=-L}^{L} q_{L, l}^{2}+\vec{\eta}^{T} \cdot \mathcal{H}\left(\vec{q}_{2}, \vec{q}_{4}\right) \cdot \vec{\eta}
$$

at fixed distortion $\overrightarrow{q_{2}}, \overrightarrow{q_{4}}$ and electron state $\vec{\eta}$. Minimization of $V$ with respect to $\vec{\eta}$ yields, by definition, the (adiabatic) BO potential. If we keep $\vec{\eta}$ fixed instead, and minimize (18) with respect to $\vec{q}=\left(\overrightarrow{q_{2}}, \overrightarrow{q_{4}}\right)$, then we get the extremal points of the BO potential in configuration space as

$$
\vec{q}=\vec{q}(\vec{\eta})
$$

Inserting (19) in (18), we obtain a function $\hat{V}$ of the electronic coordinates alone, hence a function defined on the ES, not on the space of distortion like the BO potential. We still have to ensure that the electronic vector is an eigenvector of the interaction Hamiltonian. Ceulemans [27] realized that this condition is equivalent to a second minimization procedure, on the ES; moreover, he recognized that the $\hat{V}$ function is isoextremal to the BO surface, that is, its extremal points on the ES correspond (in a 2:1 way) to extremal points of the BO surface. Then the JTM has the same symmetry properties of the set of minima of $\hat{V}$.

We apply this procedure of reverse-order minimization to the present system. We list here the distortions $q_{2}$ and $q_{4}$ which minimize $V$ at fixed $\vec{\eta}$ on the ES:

$$
q_{2,2}=-\frac{2}{\sqrt{7}} g_{2} \eta_{1} \eta_{3}-\frac{1}{2} \sqrt{\frac{3}{7}} g_{2}\left(\eta_{4}^{2}-\eta_{5}^{2}\right)
$$




$$
\begin{aligned}
q_{2,1} & =\sqrt{\frac{3}{7}} g_{2}\left(\eta_{2} \eta_{4}+\eta_{1} \eta_{5}\right)+\frac{1}{\sqrt{7}} g_{2} \eta_{3} \eta_{5} \\
q_{2,0} & =-\frac{1}{\sqrt{7}} g_{2}\left(\eta_{1}^{2}+\eta_{2}^{2}-\eta_{3}^{2}\right)+\frac{1}{2 \sqrt{7}} g_{2}\left(\eta_{4}^{2}+\eta_{5}^{2}\right) \\
q_{2,-1} & =\sqrt{\frac{3}{7}} g_{2}\left(\eta_{2} \eta_{5}-\eta_{1} \eta_{4}\right)+\frac{1}{\sqrt{7}} g_{2} \eta_{3} \eta_{4} \\
q_{2,-2} & =-\frac{2}{\sqrt{7}} g_{2} \eta_{2} \eta_{3}+\sqrt{\frac{3}{7}} g_{2} \eta_{4} \eta_{5} \\
q_{4,4} & =-\frac{1}{2} g_{4}\left(\eta_{1}^{2}-\eta_{2}^{2}\right) \\
q_{4,3} & =\frac{1}{\sqrt{2}} g_{4}\left(\eta_{2} \eta_{4}-\eta_{1} \eta_{5}\right) \\
q_{4,2} & =-\sqrt{\frac{3}{7}} g_{4} \eta_{1} \eta_{3}+\frac{1}{\sqrt{7}} g_{4}\left(\eta_{4}^{2}-\eta_{5}^{2}\right) \\
q_{4,1} & =\sqrt{\frac{1}{14}} g_{4}\left(\eta_{2} \eta_{4}+\eta_{1} \eta_{5}\right)-\sqrt{\frac{6}{7}} \eta_{3} \eta_{5} \\
q_{4,0} & =-\frac{1}{2 \sqrt{35}} g_{4}\left(\eta_{1}^{2}-\eta_{2}^{2}-6 \eta_{3}^{2}+4 \eta_{4}^{2}+4 \eta_{5}^{2}\right) \\
q_{4,-2} & =-\sqrt{\frac{3}{7}} g_{4} \eta_{2} \eta_{3}+\frac{2}{\sqrt{7}} g_{4} \eta_{4} \eta_{5} \\
q_{4,-1} & =-\sqrt{\frac{1}{14}} g_{4}\left(\eta_{1} \eta_{4}+\eta_{2} \eta_{5}\right)-\sqrt{\frac{6}{7}} \eta_{3} \eta_{4} \\
\left.q_{4} \eta_{5}\right) & =-\eta_{1} \eta_{2} \\
q_{4} & =-1
\end{aligned}
$$

We can choose a (hyper-polar) representation for the electronic coordinates such that the unitarity condition (17) is automatically satisfied

$$
\begin{aligned}
& \eta_{1}=\sin \theta_{1} \sin \theta_{2} \sin \theta_{3} \cos \theta_{4}, \\
& \eta_{2}=\sin \theta_{1} \sin \theta_{2} \sin \theta_{3} \sin \theta_{4}, \\
& \eta_{3}=\cos \theta_{1}, \\
& \eta_{4}=\sin \theta_{1} \cos \theta_{2}, \\
& \eta_{5}=\sin \theta_{1} \sin \theta_{2} \cos \theta_{3} .
\end{aligned}
$$

Here, $\theta_{i} \mathrm{i}=1,2,3$ are the azimuthal angles and $\theta_{4}$ the anomaly. Substituting (22) in (20) and (21), and then back into (18) we obtain an expression for the minimum $E_{J T}$ of $\hat{V}$ : 


$$
E_{J T}=-\frac{1}{70}\left(5 g_{2}^{2} \omega_{2}+9 g_{4}^{2} \omega_{4}\right)
$$

and for the JTM coordinates

$$
\left|\vec{q}_{2}\right|=\frac{1}{\sqrt{7}} g_{2},\left|\vec{q}_{4}\right|=\frac{3}{\sqrt{35}} g_{4} .
$$

\section{Is there a Berry phase?}

As Eq. (23) shows, $E_{J T}$ is independent of the $\eta$ parameters, and is therefore a constant on the whole ES. This is a nontrivial consequence of the large amount of geometrical freedom which is allowed to the molecule by the large degeneracy of the vibron modes: on the contrary, when the dimension of $\vec{q}$-space is less than that of the ES (like for example in the $h \otimes G$ JT system - relevant for the coupling of $h_{u}$ with the $G_{g}(3)$ mode, which has no $H_{g}$ partner), $E_{J T}$ is not flat, but is characterized by some amount of corrugation. The isoextremality property of the $\mathcal{D}^{(2)} \otimes\left(\mathcal{D}^{(2)} \oplus \mathcal{D}^{(4)}\right)$ implies that the JTM, must have locally the same structure as the ES, here $S^{4}$, a sphere in five dimensions.

However, upon closer scrutiny, we note a topological difference between the ES and the JTM, due to the 2:1 correspondence mentioned above. In particular, the ES is a projective space, as opposite points on the sphere correspond to the same point in the $q$-coordinates space (they belong to the same "ray"). This is equivalent to the fact that normalized eigenvectors of a symmetric matrix are defined up to a sign. The JTM does not share this property, since there are no pairs of points on the JTM that generate the same $\vec{\eta}$. Here is the source of the Berry phase: to each path in the JTM there corresponds a path on the ES, but the two are topologically different. In particular, a loop in the JTM may correspond either to a closed path, or to a path from a point to its opposite (antipode) on the ES. In general, the latter path cannot be reduced to a point, and there will be a topological effect (Berry phase). This fact is illustrated in Fig. 1 on an ordinary $S_{2}$ sphere, in 3-dimensional space, representing the ES. The line $\Gamma$ corresponds to a closed loop on the JTM: there is no way to make point $B$ coincide with point $A$ in such a way that then we can just shrink the 
path and have it coincide to a trivial one such as $\Gamma_{1}$. However, we are going to encounter situations where apparently nontrivial loops become shrinkable to a point via singularities in the mapping between the ES and the JTM.

Of the most general set of coupling parameters, we consider three special cases: (a) $\mathcal{D}^{(2)} \otimes \mathcal{D}^{(2)}$, i.e. $g_{2} \neq 0, g_{4}=0$; (b) $\mathcal{D}^{(2)} \otimes \mathcal{D}^{(4)}$, i.e. $g_{2}=0, g_{4} \neq 0$; and finally (c) $\mathcal{D}^{(2)} \otimes\left(\mathcal{D}^{(2)} \oplus \mathcal{D}^{(4)}\right)$, i.e. $\omega_{2}=\omega_{4}, g_{2}=g_{4} \neq 0$.

(a) $\mathcal{D}^{(2)} \otimes \mathcal{D}^{(2)}$. Here, the $\vec{q}-\vec{\eta}$ mapping is given by Eq. (20) and (22). A class of closed paths on $q$-manifold does, as always, transport $\vec{\eta}$ into its antipode $-\vec{\eta}$. However, we find in this case that a whole ("equatorial") line joining two antipodes on the ES, defined by $\theta_{1}=\theta_{2}=\theta_{3}=\pi / 2$ (i.e. $\eta_{1}^{2}+\eta_{2}^{2}=1, \eta_{3}=\eta_{4}=\eta_{5}=0$ ) and parameterized by $\theta_{4}=0 \rightarrow \pi$, in fact corresponds to a single point $\vec{q}_{2}=\left(0,0,-\frac{g_{2}}{\sqrt{7}}, 0,0\right)$ in $q$-space. The antipodes are therefore topologically joined, and there are no loops on the JTM that cannot be deformed to a point. Hence, there is no Berry phase in this case.

(b) $\mathcal{D}^{(2)} \otimes \mathcal{D}^{(4)}$. The $\vec{q}-\vec{\eta}$ mapping is now through Eq. (21) and (22). A closed loop on the JTM transports $\vec{\eta}$ into $-\vec{\eta}$ if it encircles a conical intersection in configuration space. This is clearly the case of the line described in (a), here corresponding to

$$
\begin{aligned}
q_{4,4} & =-\frac{1}{2} g_{4} \cos \left(2 \theta_{4}\right) \\
q_{4,0} & =-\frac{1}{2 \sqrt{35}} g_{4} \cos \left(2 \theta_{4}\right) \\
q_{4,-4} & =-\frac{1}{2} g_{4} \sin \left(2 \theta_{4}\right)
\end{aligned}
$$

Now only the $\vec{q}$ antipodes are topologically equivalent, while the loops that join them are nontrivial: there is a Berry phase of $\pi$ in the electronic transport in this case.

(c) $\mathcal{D}^{(2)} \otimes\left(\mathcal{D}^{(2)} \oplus \mathcal{D}^{(4)}\right)$. In this case the $\vec{q}-\vec{\eta}$ mapping passes through both Eq. (20) and Eq. (21). The Berry phase of case (b) is therefore conserved upon addition of the $\mathcal{D}^{(2)}$ mode. This case has an instructive analogy to simpler JT-coupled systems. 


\section{D. "Propagation" of Berry phases}

In case (c) above, we add to a Berry-phase entangled DJT system an additional $L=2$ vibron mode, also coupled to the same electronic state. It is interesting to consider what happens to the Berry phase when the added mode, on its own, carries no Berry phase. The enlargement of the $\vec{q}$-space introduces a large freedom for creating new loops in $\vec{q}$. One might in principle think that some of these loops could manage to avoid the conical intersections of $\mathrm{BO}$ levels, thus healing the connectivity properties of the JTM, and short-circuiting out the Berry phase. We found instead that this is not the case, and $\mathcal{D}^{(2)} \otimes\left(\mathcal{D}^{(2)} \oplus \mathcal{D}^{(4)}\right)$ retains the Berry phase of $\mathcal{D}^{(2)} \otimes \mathcal{D}^{(4)}$.

An example of analogous situation is the $t \otimes(T \oplus E)$ problem, extensively studied in the literature [15, 16] : the $t \otimes T$ problem is Berry-phase-entangled, while the $t \otimes E$ is not. It is well known that $t \otimes(T \oplus E)$ problem retains the same topological properties, including the Berry phase of $\pi$, of the $t \otimes T$ system, the effect of the $E$ mode consisting essentially only in flattening the JTM, and removing inter-minima barriers.

The reason why new loops seem to be ineffective in destroying the Berry phase in the total vibron space is that this space is the cartesian product of the single-mode spaces: the degeneracy points, opening "holes" in the space of the first vibron, propagate (locally, at least) in the global space along the second-vibron directions. The global conservation of connectivity in the whole product space is a nontrivial topological problem, whose general outcome is not known to us. In both examples above, we simply note that things go as if the Berry phase carried by the first mode is strictly conserved.

In the present situation, the coupled $\mathcal{D}^{(2)}$ vibrational mode does not present any topological effect (a), whereas the $\mathcal{D}^{(4)}$, as seen in case (b), carries a Berry phase. Thus the

overall $\mathcal{D}^{(2)} \otimes\left(\mathcal{D}^{(2)} \oplus \mathcal{D}^{(4)}\right)$ system is affected by topological effects, and this remains true even for different couplings $g_{2} \neq g_{4} \neq 0$.

The fullerene cations provide a third example of this behavior. The $\mathcal{D}^{(2)} \otimes \mathcal{D}^{(4)}$ is naturally resolved in its icosahedral composition $h \otimes G$ plus $h \otimes H$. In the light of the above 
considerations, we expect that in this system the Berry phase should be carried by the $G$ mode [28]. That this is indeed the case, can thus be seen as a natural consequence of the absence of Berry phases in $h \otimes H$ (i.e. $\left.\mathcal{D}^{(2)} \otimes \mathcal{D}^{(2)}\right)$, and its presence in $\mathcal{D}^{(2)} \otimes \mathcal{D}^{(4)}$.

\section{SEMICLASSICAL QUANTIZATION AND THE EFFECTS OF THE BERRY PHASE}

So far we have treated the ions as classical. We study here the consequences of the discussed symmetries of the JTM and of the presence/absence of the Berry phase on the quantum spectrum of the coupled system, by quantizing the semiclassical ionic motion. The general form of the ion kinetic energy is

$$
H_{0}=-\sum_{L=2,4} \frac{\hbar \omega_{L}}{2} \sum_{l=-L}^{L} \frac{\partial^{2}}{\partial q_{L, l}^{2}} .
$$

It is convenient to use the classical language for the derivation of the kinetic operator restricted to the semiclassical region:

$$
K=\frac{1}{2} \sum_{L=2,4} \frac{\hbar}{\omega_{L}} \sum_{l=-L}^{L} \dot{q}_{L, l}^{2} .
$$

In the semiclassical limit, valid when $g_{L}$ are very large, the ionic motion is confined to the JTM, itself given by Eq. (24). Hence, only the tangential part of the kinetic energy (26) is relevant, which, upon introduction of $\zeta=\frac{\omega_{2}}{\omega_{4}}-1$ can be written

$$
K=\frac{\hbar}{2 \omega_{2}} \sum_{i j} \dot{\theta}_{i} G^{i j} \dot{\theta}_{j}
$$

where $G^{i j}$ is the metric induced on the JTM by the electronic parametrization:

$$
G^{i j}=\sum_{L=2,4}\left(1+\delta_{L, 4} \zeta\right) \sum_{l=-L}^{L} \partial_{\theta_{i}} q_{L, l} \partial_{\theta_{j}} q_{L, l}
$$

Not much progress is possible in this form, due to the general low-symmetry of the kinetic term. However, in the special case of equal frequencies $\left(\omega_{2}=\omega_{4}=\omega: \zeta=0\right)$ and equal couplings, where the true symmetry of the problem is $\mathrm{SO}(5)$ 23], the metric is rewritten as: 


$$
G^{i j}=\partial_{\theta_{i}} \vec{q} \partial_{\theta_{j}} \vec{q}
$$

Thus, the kinetic energy corresponds to that of a free particle on a sphere in 5 dimensions:

$$
K=I \frac{\hbar}{\omega_{2}}\left({\dot{\theta_{1}}}^{2}+\sin \theta_{1}^{2}{\dot{\theta_{2}}}^{2}+\sin \theta_{1}^{2} \sin \theta_{2}^{2}{\dot{\theta_{3}}}^{2}+\sin \theta_{1}^{2} \sin \theta_{2}^{2} \sin \theta_{3}^{2} \dot{\theta}_{4}^{2}\right) .
$$

$I$ is the 'inertial momentum' of the system

$$
I=|\vec{q}|^{2}=\frac{2}{5} g^{2}
$$

The corresponding Laplace-Beltrami operator (describing the free motion of a quantum particle on the manifold parametrized by the $\theta_{i}$ variables) is given by the formula [29]

$$
\Delta=\frac{1}{\sqrt{G}} \partial_{\theta_{i}} G^{i j} \sqrt{G} \partial_{\theta_{j}}
$$

where $G$ is the absolute value of the determinant of the metric tensor. This operator is the usual representation of the quadratic Casimir operator of $\mathrm{SO}(5)$ on the coset manifold $\mathrm{SO}(5) / \mathrm{SO}(4)$, which is actually the sphere $S^{4}$ : this is a consequence of the exact $\mathrm{SO}(5)$ symmetry of the equal-coupling case.

The particle moves freely on the JTM, with harmonic oscillations orthogonal to it. The corresponding energy levels are therefore

$$
E=E_{J T}+\frac{\hbar^{2}}{2 I} l(l+3)+\hbar \omega \sum_{\gamma=1}^{10}\left(n_{\gamma}+\frac{1}{2}\right)
$$

$E_{J T}$ is the static JT energy (23). The last term is due to the vibrations orthogonal to the JTM. The $l(l+3)$ term corresponds to the eigenvalues of the Laplace-Beltrami operator on the sphere; $l$ is the label of the angular hyperharmonics on $S^{4}$, that realize the $[l, 0]$ representations of $\mathrm{SO}(5)$, whose degeneracy is given by

$$
N_{l}=\frac{(2 l+3)(l+2)(l+1)}{6} .
$$

The Berry phase imposes precise selection rules on these levels: since the one-hole state changes sign after a closed loop around the JTM, and the overall wave function needs to be single-valued, also the vibrational wave function has to change sign. Odd- $l$ states have 
this properties, and these are the allowed states that survive the Berry phase selection rule. This implies that the ground state is $l=1$ (fivefold degenerate) and the first excited state is $l=3$ (30-fold degenerate). Results of exact diagonalization on a truncated basis [8,30,11]

$$
\Psi=\sum a_{0 \alpha} c_{\alpha}^{\dagger}|0\rangle+a_{i \alpha} b_{i}^{\dagger} c_{\alpha}^{\dagger}|0\rangle+a_{i j \alpha} b_{i}^{\dagger} b_{j}^{\dagger} c_{\alpha}^{\dagger}|0\rangle+\ldots
$$

shown in Fig. 2, confirm the spectral structure described here for the semiclassic limit. At all values of the coupling $g$, as expected, the degeneracies are those characteristic of $\mathrm{SO}(5)$. We have done also numerical diagonalizations in the more general case of different coupling and/or frequencies: the spectra, as expected, display lower - $\mathrm{SO}(3)$ - symmetry, but a qualitatively similar behavior.

\section{A. Two holes in spin-singlet state}

In the spin-singlet $n=2$ holes case, ${ }^{1} \mathrm{C}_{60}^{2+}$ we find two main differences with the $n=1{ }^{2} \mathrm{C}_{60}^{+}$

case. First, the average distortion $|\vec{q}|=2 \sqrt{\frac{2}{5}} g$ is twice as large as in the $n=1$ case, $I$ and the $E_{J T}$ energy being 4 times as large. Secondly, and most importantly, there is a change in the selection rules. The two holes live in the same single-hole eigenvector of $\mathcal{H}\left(\vec{q}_{2}, \vec{q}_{4}\right)$, the electronic wave function being the product of two equivalent eigenfunctions for the space coordinates and an antisymmetric part for the spin degrees of freedom. Since each hole state changes sign after a closed loop in the JTM, the overall hole state does not. Thus the vibrational wave function must also be single-valued in order to have an overall single-valued wave function for the system. As a consequence, only even-l states survive: in particular, the ground state is $l=0$ (non-degenerate) and the first excited state is $l=2$ (14-fold degenerate). Exact diagonalization results, shown in Fig. 2(b), confirm that the symmetry and, to a certain degree the spectral structure described here for the semiclassic limit are retained in the weak-coupling limit. 


\section{B. The $2 \otimes(2 \oplus 4)$ unequal couplings case}

In the more general non-equal coupling case $g_{2} \neq g_{4}$, including also the $g_{2}=0$ case, we could try to use the metric as for the equal coupling in order to obtain an analogous expression for the kinetic energy. Unfortunately, when the couplings are different, we do not get a treatable expression like (31), and we cannot follow the same route for quantizing the system.

We recall instead that the structure of the JTM is the same as that of ES, and in particular that $\mathrm{SO}(5)$ symmetry is retained. The expression of the minimum potential energy (23) suggests a flat JT trough, for any values of the frequencies and couplings. Therefore, it is likely that the free motion of the particle on the JTM is described again by the quadratic Casimir operator of $\mathrm{SO}(5)$, retaining for the low-energy excitations a spectral structure similar to Eq. (34).

However, although the full $\mathrm{SO}(5)$ symmetry is retained in the $g \rightarrow \infty$ semiclassical limit, for any non-infinite value of the couplings, the $\mathrm{SO}(5)$ representations will be split into representations of the $\mathrm{SO}(3)$ group [31]. From this point of view we can see the differences between the frequencies and between the coupling strengths as symmetry-reducing perturbations, that become effective when moving away from the strong-coupling limit.

\section{The $2 \otimes 2$ case}

This case, corresponding to $h_{u} \otimes H_{g}$ in a $\mathrm{C}_{60}^{+}$cation, is very interesting because of the peculiar features described above, i.e. absence of topological singularities and, in particular, absence of a Berry phase. Furthermore, this case is worth additional analysis, since it provides an alternate limit of our approach for the different coupling case (of which this is an extreme case).

We treat again the system as a quantization problem on a five-dimensional sphere. In this case, it is possible to find the extremal eigenvalues of the interaction matrix $\mathcal{H}\left(\vec{q}_{2}\right)=$ 
$\frac{1}{2} g_{2} \hbar \omega_{2} \tilde{B}_{2}$, as solutions of the characteristic polynomial equation for $\tilde{B}_{2}$ :

$$
\lambda^{5}-\left|\vec{q}_{2}\right|^{2} \lambda^{3}+7 f_{0}\left(\vec{q}_{2}\right) \lambda^{2}+\frac{12}{49}\left|\vec{q}_{2}\right|^{4} \lambda-4 f_{0}\left(\vec{q}_{2}\right)\left|\vec{q}_{2}\right|^{2}=0
$$

where

$$
\begin{aligned}
f_{0}\left(\vec{q}_{2}\right)= & -\frac{1}{49 \sqrt{7}}\left[3 q_{2,0}\left(q_{2,1}^{2}+q_{2,-1}^{2}\right)-6 q_{2,0}\left(q_{2,2}^{2}+q_{2,-2}^{2}\right)\right. \\
& \left.+2 q_{2,0}^{3}+3 \sqrt{3} q_{2,2}\left(q_{2,1}^{2}-q_{2,-1}^{2}\right)-6 \sqrt{3} q_{2,1} q_{2,-1} q_{2,-2}\right] .
\end{aligned}
$$

This polynomial can be decomposed as

$$
\left(\lambda^{2}-\frac{4}{7}\left|\vec{q}_{2}\right|^{2}\right)\left[\lambda^{3}-\frac{3}{7}\left|\vec{q}_{2}\right|^{2} \lambda+7 f_{0}\left(\vec{q}_{2}\right)\right]=0
$$

The extremal eigenvalues of $\tilde{B}_{2}$ are

$$
\lambda_{1 / 5}=\mp \frac{2}{\sqrt{7}}\left|\vec{q}_{2}\right|
$$

in accord with the $\mathrm{SO}(5)$ symmetry of the JTM in the 5-dimensional $\mathcal{D}^{(2)}$ space. The three remaining intermediate eigenvalues can be expressed as

$$
\begin{aligned}
& \lambda_{2}=\left|\vec{q}_{2}\right| \frac{2}{\sqrt{7}} \cos \left(\frac{\Psi}{3}-\frac{2 \pi}{3}\right) \\
& \lambda_{3}=\left|\vec{q}_{2}\right| \frac{2}{\sqrt{7}} \cos \left(\frac{\Psi}{3}\right) \\
& \lambda_{4}=\left|\vec{q}_{2}\right| \frac{2}{\sqrt{7}} \cos \left(\frac{\Psi}{3}+\frac{2 \pi}{3}\right)
\end{aligned}
$$

where

$$
\cos \Psi=-49 \sqrt{7} \frac{f_{0}\left(\overrightarrow{q_{2}}\right)}{2\left|\vec{q}_{2}\right|^{3}} .
$$

In order to obtain the eigenenergies of $\mathcal{H}\left(\vec{q}_{2}\right)$ one has to multiply these eigenvalues by a factor $\frac{1}{2} g_{2} \hbar \omega_{2}$. By inspection, $\lambda_{2,3,4}$ are bound in the $\left[\lambda_{1}, \lambda_{5}\right]$ interval. However, it is possible to find many points on the JTM such that $\lambda_{1}=\lambda_{2}$, for example the "south pole" $q_{0} /|\vec{q}|=-1$ and a one-dimensional manifold on the "equator" $q_{0}=0$. Since $\lambda_{1}$ is constant and $\lambda_{2}$ is analytical in $\overrightarrow{q_{2}}$, in particular around the tangency points where the two eigenvalues get 
degenerate, these degeneracies are not conical intersections of the two adiabatic surfaces. The two surfaces have therefore just a contact of second order at these points. The absence of conical intersections implies the absence of any topological effect [3].

Because of the absence of a Berry phase, already discussed in Sect. III], the strong coupling behavior of the system, described in terms of the $\mathrm{SO}(5)$ representations, is such that we expect a $[0,0]$ (non degenerate) ground state, a [1,0] (fivefold degenerate) first excited state, a $[2,0]$ (14-fold degenerate) second excited state, and so on. Moreover, absence of topological effects makes the two-holes case not essentially different from the one-hole problem in the strong coupling limit. Of course, the static JT energy and inertial momentum is just four times larger than in the one hole case, but the level sequence in the strong-coupling limit is the same.

Coming back to the single-hole case, let us now move away from the strong-coupling limit, to discuss the more general case of an arbitrary coupling $g_{2}$. We might naïvely expect, at first, the ground state symmetry to be preserved for arbitrary $g_{2}$, as was the case in other DJT problems examined previously [8]. However, a closer inspection reveals that this is not true, and rather that there must be a level crossing as a function of $g_{2}$. A first indication comes from the fact that the ground state must in fact be $L=2$, i.e. fivefold degenerate, as $g_{2} \rightarrow 0$, reflecting just the uncoupled hole $\left(h_{u}\right)$ degeneracy. A second hint comes from the tangency of the lowest with the second BO surface, noted above. Could this tangency have a role in the expected level crossing from $L=2$ at $g \rightarrow 0$ to $L=0$ at $g \rightarrow \infty$ ? To study this possibility, we resort to numerical diagonalization of Hamiltonian (3) on a truncated basis (36), for finite values of $g_{2}$. As pointed out above, for finite coupling the symmetry of the system is strictly $\mathrm{SO}(3)$. Therefore, we expect the large degeneracies characteristic of $\mathrm{SO}(5)$ symmetry to build up from converging $\mathrm{SO}(3)$ states for large $g_{2}$. The $[0,0]$ and $[1,0]$ states map directly on the $\mathcal{D}^{(0)}$ and $\mathcal{D}^{(2)} \mathrm{SO}(3)$ representations respectively [31], while the $\mathrm{SO}(3)$ composition of $[2,0]$ is $\mathcal{D}^{(2)} \oplus \mathcal{D}^{(4)}$. Therefore, we seek the convergence of two such levels in the strong-coupling limit.

The numerical solution of the 1-hole problem (see Fig. 3(a)), shows indeed the correct 
order of the levels for large couplings $\left(g_{2} \gtrsim 8\right)$, whereas for $g_{2} \leq \sim 8$ the $[0,0]$ and $[1,0]$ levels cross and become inverted. This level inversion can in fact be precisely seen as a consequence of the tangency of the two adiabatic potential surfaces described above. The two $\mathrm{BO}$ sheets lie close in a significant region of configuration space. Treating the upper sheet as a perturbation for the eigenstates of the lower sheet, the energy of all these states is pushed up by this correction. The $l=0$ (isotropic) states feel more efficiently this perturbation than the $l>0$ hyperspherical harmonics, since they can arrange their nodes so as to avoid the tangency line. In conclusion, the effect of the tangency is to increase the energy of the $l=0$ level more than that of $l=1$, so that, for coupling not too large, $g \gtrsim 8$, the latter is the ground state.

In the 2-holes (spin singlet) case (Fig. B(b)) this inversion is not present, the ground state being nondegenerate $\left(\mathcal{D}^{(0)}\right)$ for any coupling $g_{2}$. Again, from a weak-coupling approach, this is a simple consequence of the order of splitting of the $\mathrm{d}^{2}$-fermion spin-singlet states upon perturbative JT coupling with a $\mathcal{D}^{(2)}$ vibration, that is (from lowest to highest) $\mathcal{D}^{(0)}, \mathcal{D}^{(4)}$, $\mathcal{D}^{(2)}$. From the strong-coupling side, the only observed crossover happens between the $\mathcal{D}^{(2)}$ and $\mathcal{D}^{(4)}$ low excitations above the ground-state. This means that the BO picture of the two holes in the same single-hole orbital, although appropriate to the strong-coupling limit, is rather poor for intermediate JT coupling, when the two fermions manage to arrange in a cooperative state preserving the global $\mathcal{D}^{(0)}$ symmetry despite the tangency of the lowest BO sheets.

\section{The $h_{u} \otimes G_{g}$ case}

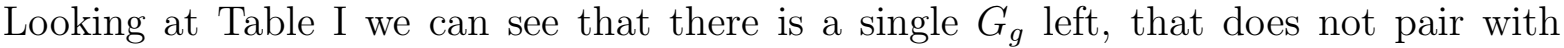
any $H_{g}$ mode. We must therefore treat the coupling of mode $G_{g}(3)$ according to the true icosahedral picture. Actually the static $h \otimes G$ JT problem has been treated in the literature [32]. The set of absolute minima of the system if made up of ten isolated points in the four-

dimensional $G_{g}$ space. Tunnel splitting among these ten $\mathrm{BO}$ valleys give rise to vibronic 
states of symmetry $A, G$, and $H$. In Ref. 28] the diagonalization of the corresponding matrix has been performed including the proper provision for the Berry phase. The result is that the $H$ quintet is the true ground state, followed by the $G$ quartet and the $A$ singlet in the order. Thus, the Berry phase again acts in such a way to enforce a fivefold degenerate ground state all the way from weak to strong coupling, in analogy with the $h \otimes(G \oplus H) \mathrm{JT}$ problem, and in contrast to the $h \otimes H$ case discussed in Sect. IV G. This confirms that, as anticipated, the Berry phase in the $h \otimes(G \oplus H)$ JT problem comes from $h \otimes G$ sector.

\section{DISCUSSION}

We have studied the symmetry aspects and the possible presence of a Berry phase in $\mathrm{C}_{60}^{+}$ and $\mathrm{C}_{60}^{2+}$ in the linear and spherical approximation. We find that while ${ }^{1} \mathrm{C}_{60}^{2+}$ has no Berry phase, ${ }^{2} \mathrm{C}_{60}^{+}$does, and is very rich and intriguing.

Clearly, the present analysis is only a first step. In reality, fullerene is not a sphere, but a discrete icosahedron, and the $\mathcal{D}^{(4)}$ modes are split into $H_{g} \oplus G_{g}$ modes. Nonetheless the real shape of the molecule can be seen as a perturbation on the spherical picture (Tab. 四): the $\mathrm{SO}(5)$ and $\mathrm{SO}(3)$ levels are split but the selection rules are still present. The ÖpikPryce approach can still be applied in order to obtain the JTM in icosahedral symmetry [32]. Quite generally the BO potential will warp in such a way to originate a discrete set of minima, instead of a continuous 4-dimensional manifold. The molecular system will execute tunneling among these minima. However, as shown by Ham 33], the Berry phase imposes the degeneracy of the ground state (irrespective of the presence and height of barriers between the BO minima), which still is fivefold degenerate $\left(\mathcal{D}^{(2)} \rightarrow H\right)$. Therefore, we expect the ground state symmetry to be ${ }^{2} H_{u}$ for real $\mathrm{C}_{60}^{+}$, which has a Berry phase, and ${ }^{1} A_{g}$ for singlet $\mathrm{C}_{60}^{2+}$, which does not.

A quantitative study of the properties of $\mathrm{C}_{60}^{+}$is clearly beyond the scope of the present work. In order to accomplish that, a full set of e-v couplings $g_{H_{g}(i)} i=1, \ldots 8$ and $g_{G_{g}(i)} i=$ $1, \ldots 6$ will have to be known (either from calculations, or fit to experiments), and inserted 
into a diagonalization procedure similar to that previously carried out in the case of $\mathrm{C}_{60}^{n-}$ 8, 30,11]. While that remains to be done, we can list, for the time being, some tentative physical implications of our results.

(i) Low-energy electron detachment. In a way analogous to that proven for the case of $\mathrm{C}_{60}^{-}$, one can surmize that $\mathrm{C}_{60}^{+}$cannot have any $A_{g}$ low-energy states. It follows that if an electron is detached from $\mathrm{C}_{60}$ into a low-energy state, the s-wave cross-section will be zero. and there will be a centrifugal barrier in detachment too, similar in nature to that pointed out for attachment [10]. This effect could be related to the thermoionic anomalies pointed out by Yeretzian et al. 34

(ii) Low-energy tunneling excitation. In the spherical approximation, the lowest excitation of $\mathrm{C}_{60}$ is a 30 -fold degenerate $\mathrm{SO}(5)$ " $l=3$ " tunneling multiplet, as required by the Berry phase. Split, as it will be by the lower $\mathrm{SO}(3)$ symmetry, as well as by the true $\mathcal{I}_{h}$ effects, this multiplicity might still be identifiable spectroscopically.

(iii) $C_{60}$ photoemission spectrum. In the valence photoemission spectrum of $\mathrm{C}_{60} \rightarrow$ $\mathrm{C}_{60}+e_{\vec{k}}^{-}$the electron kinetic energy distribution contains very direct information - in the so-called final state approximation - about vibron and hole-vibron coupling in $\mathrm{C}_{60}^{+}$. The outgoing electron shakes up multi-vibron excitations of $\mathrm{C}_{60}^{+}$, with a relative probability which can be calculated in principle, in a manner similar to that used by Gunnarsson 30 for $\mathrm{C}_{60}^{-}$. Very recent data [35] indicate that these shake-ups do exist and are in fact stronger for $\mathrm{C}_{60}$ than for $\mathrm{C}_{60}^{-}$.

We hope to return to these topics in subsequent work.

\section{ACKNOWLEDGMENTS}

P. De Los Rios is grateful to S. Panzeri for many useful discussions. We acknowledge exchange of information and discussion with P. Brühwiler and F. Iachello. This work was partly sponsored by NATO, through CRG 920828, and by EEC through ERBCHRXCT 940438. 


\section{REFERENCES}

[1] R. Englman, The Jahn Teller Effect in Molecules and Crystals (Wiley, London, 1972).

[2] H. C. Longuet-Higgins, U. Öpik, M.H.L. Pryce, and R.A. Sack, Proc. Roy. Soc. A 244, 1 (1958).

[3] H. C. Longuet-Higgins, Adv. Spect. 2 429, (1961), and references therein; G. Herzberg and H. C. Longuet-Higgins, Discuss. Faraday Soc. 35, 77 (1963); H. C. Longuet-Higgins, Proc. Roy. Soc. London A 344, 147 (1975); C. A. Mead and D. G. Truhlar, J. Chem. Phys. 70, 2284 (1979).

[4] U. Öpik and M.H.L. Pryce, Proc. Roy. Soc. A 238, 425 (1957).

[5] M. V. Berry, Proc. R. Soc. Lond. A 392, 45 (1984).

[6] G. Delacrétaz, E. R. Grant, R. L. Whetten, L. Wöste, and J. W. Zwanziger, Phys. Rev. Lett. 56, 2598 (1986).

[7] C. A. Mead, Rev. Mod. Phys. 64, 51 (1992).

[8] A. Auerbach, N. Manini, and E. Tosatti, Phys. Rev. B 49, 12998 (1994).

[9] N. Manini, E. Tosatti, and A. Auerbach, Phys. Rev. B 49, 13008 (1994).

[10] E. Tosatti, and N. Manini, Chem. Phys. Lett. 223, 61 (1994).

[11] N. Manini and E. Tosatti, in Recent Advances in the Chemistry and Physics of Fullerenes and Related Materials: Volume 2, edited by K.M. Kadish and R.S. Ruoff (The Electrochemical Society, Inc., Pennington-NJ, 1995).

[12] N. Manini, E. Tosatti, and S. Doniach, Phys. Rev. B 51, 3731 (1995).

[13] G. Santoro, M. Airoldi, N. Manini, E. Tosatti, and A. Parola Phys. Rev. Lett. 74, 4039 (1995).

[14] G. Santoro, N. Manini, A. Parola, and E. Tosatti, submitted to Phys. Rev. B. 
[15] M.C.M. O’Brien, Phys. Rev. 187, 407 (1969).

[16] M.C.M. O’Brien, J. Phys. C 4, 2524 (1971).

[17] S. Satpathy, Chem. Phys. Lett. 130, 545 (1986).

[18] M. Ozaki, and A. Takahashi, Chem. Phys. Lett. 127, 242 (1986).

[19] M.R. Savina, L.L. Lohr, and A.H. Francis, Chem. Phys. Lett. 205, 200 (1993).

[20] S. Cesare, and V. Del Duca, Riv. Nuovo Cimento 10, 1 (1987).

[21] S.L. Altmann and P. Herzig, Point-Group Theory Table (Oxford Science Pub., Oxford, 1994).

[22] A. Ceulemans, P.W. Fowler, and I. Vos, J. Chem. Phys. 100, 5491 (1994).

[23] D.R. Pooler, J. Phys. C 12, 1029 (1980).

[24] M. Hamermesh, Group Theory and its applications to physical problems (AddisonWesley, London, 1962), Ch. 10.

[25] Of course, this very high symmetry breaks down as soon as the $\mathcal{D}^{(2)}$ and $\mathcal{D}^{(4)}$ modes are no more degenerate and/or equally coupled, reducing the symmetry to the expected $\mathrm{SO}(3)$.

[26] J.F. Cornwell, Group Theory in Physics, Vol. II (Academic Press, New York, 1984).

[27] A. Ceulemans, J. Chem. Phys. 87, 5374 (1987).

[28] J. P. Cullerne, M. N. Angelova and M. C. M. O'Brien, J. Phys.: Condens. Matter 7, 3247 (1995).

[29] A.O. Barut and R. Raczka, Theory of Group Representations and Applications (World Scientific, Singapore, 1986) p. 302-309.

[30] O. Gunnarsson, H. Handschuh, P.S. Bechthold, B. Kessler, G. Ganteför, and W. Eber- 
hardt, Phys. Rev. Lett. 74, 1875 (1995); O. Gunnarsson, Phys. Rev. B 51, 3493 (1995).

[31] F. Iachello and A. Arima, The interacting boson model (Cambridge, New York, 1987), Ch. 2 .

[32] A. Ceulemans, and P.W. Fowler, J. Chem. Phys. 93, 1221 (1990).

[33] F. S. Ham, Phys. Rev. Lett. 58, 725 (1987); F.S. Ham, J. Phys.: Condens. Matter 2, 1163-1177 (1990).

[34] C. Yeretzian, K. Hansen and R.L. Whetten, Science 260, 652 (1993).

[35] P. Brühwiler, private communication.

[36] J. Kohanoff, W. Andreoni, and M. Parrinello, Chem. Phys. Lett. 198, 472 (1992); J. Kohanoff, PhD Thesis No. 10079 ETH Zurich (1993); W. Andreoni, in Electronic Properties of New Materials: Fullerenes, Proceedings of the Kirchberg Winter School, edited by H. Kuzmany, J. Fink, M. Mehring and S. Roth, March 6-11, 1993 (Springer Verlag, Berlin, 1994). 


\section{FIGURES}

FIG. 1. A three-dimensional representation of the (truly five-dimensional) electronic sphere (ES): the path $\Gamma$ connects opposite points (antipodes), which correspond to the same point on the JTM. Thus $\Gamma$ maps, through Eqs. (20) and (21), on a closed loop in the JTM. This class of loops is usually entangled with a nonzero Berry phase, while trivial loops, such as $\Gamma_{1}$ can be smoothly deformed to one point, with associated zero Berry phase. However, in the case $g_{4}=0$, due to the singularity of mapping (20), a whole equatorial line $\Lambda$ maps to a single $\vec{q}$-point. In this special case, point B identifies with point A on the JTM, thus $\Gamma$ becomes equivalent to $\Gamma_{1}$, and no Berry phase is possible.

FIG. 2. The low-lying states of the spectrum of the $L=2 \otimes(L=2 \oplus L=4)([1,0] \otimes[2,0]$, in $\mathrm{SO}(5)$ notation) JT system, as a function of the coupling $g=g_{2}^{2}=g_{4}^{2}$. Symmetry labels are indicated according to the $\mathrm{SO}(5)$ group representations. The truncated basis includes up to 7 vibrons. Panel (a): the levels originated from the 0 - and 1-vibron states, for $n=1$ holes (see also Table I for the small- $g$ limit). Panel (b): the three levels originating from the 0 -vibrons multiplet for $n=2$ holes. The tenfold-degenerate $[0,2]$ orbital state is in a triplet spin configuration.

FIG. 3. The low-lying states of the spectrum of the $L=2 \otimes L=2$ coupled JT system as a function of the coupling $g_{2}^{2}$. We include in the diagonalization states with up to 35 vibrons. For $n=1$ fermions included (panel a), the reference level is taken as the lowest $L=2$ state, that is also

the ground state for $g_{2}<\sim 8$. For $n=2$ fermions included (panel b) the energies are excitations above the $L=0$ ground state. Only spin-singlet states are plotted. 


\section{TABLES}

\begin{tabular}{|c|c|c|c|}
\hline $\begin{array}{c}\mathrm{SO}(3) \\
\quad L\end{array}$ & $\mathcal{I}_{h}$ & $\begin{array}{c}\text { Calculated 22 } \\
\mathrm{cm}^{-1}\end{array}$ & $\begin{array}{c}\text { Calculated 36] } \\
\mathrm{cm}^{-1}\end{array}$ \\
\hline \multirow{2}{*}{2} & $H_{g}(1)$ & 214 & 261 \\
\hline & $H_{g}(4)$ & 727 & 775 \\
\hline 3 & $G_{g}(3)$ & 557 & 594 \\
\hline \multirow{4}{*}{4} & $H_{g}(2)$ & 387 & 435 \\
\hline & $G_{g}(1)$ & 374 & 482 \\
\hline & $H_{g}(6)$ & 1180 & 1208 \\
\hline & $G_{g}(4)$ & 774 & 1047 \\
\hline 5 & $H_{g}(5)$ & 1091 & 1098 \\
\hline \multirow{4}{*}{6} & $H_{g}(3)$ & 516 & 730 \\
\hline & $G_{g}(3)$ & 623 & 781 \\
\hline & $H_{g}(7)$ & 1477 & 1394 \\
\hline & $G_{g}(5)$ & 1412 & 1314 \\
\hline \multirow{2}{*}{7} & $H_{g}(8)$ & 1617 & 1573 \\
\hline & $G_{g}(6)$ & 1694 & 1479 \\
\hline
\end{tabular}

TABLE I. Experimental energies of the JT-active $G_{g}$ and $H_{g}$ modes, organized according to their spherical parentage [22]. The numbers in parenthesis in the second column are the position of the mode in order of increasing energies. 


\begin{tabular}{|c|c|c|c|c|}
\hline $\begin{array}{c}\mathrm{SO}(3) \\
L\end{array}$ & $\frac{\Delta E}{g_{2}^{2}}$ & $\frac{\Delta E}{g_{4}^{2}}$ & $\frac{\Delta E}{g^{2}}$ & $\begin{array}{r}\mathrm{SO}(5) \\
{[l, 0]}\end{array}$ \\
\hline $2^{(G S)}$ & $-\frac{1}{4}$ & $-\frac{9}{20}$ & $-\frac{7}{10}$ & {$[1,0]$} \\
\hline 0 & $-\frac{1}{2}$ & $-\frac{9}{20}$ & \multirow{6}{*}{$-\frac{19}{20}$} & \multirow{6}{*}[3,0]{} \\
\hline $3^{\dagger}$ & $-\frac{11}{28}$ & $-\frac{121}{280}$ & & \\
\hline $4^{\ddagger}$ & $-\frac{9}{28}$ & $-\frac{141}{280}$ & & \\
\hline 6 & $-\frac{1}{4}$ & $-\frac{7}{10}$ & & \\
\hline 1 & $-\frac{1}{8}$ & $-\frac{9}{20}$ & & \\
\hline $2^{*}$ & $-\frac{1}{4}$ & $-\frac{9}{20}$ & & \\
\hline $3^{\dagger}$ & $-\frac{1}{4}$ & $-\frac{9}{20}$ & \multirow[t]{3}{*}{$-\frac{23}{40}$} & \multirow[t]{3}{*}[1,2]{} \\
\hline $4^{\ddagger}$ & $-\frac{1}{4}$ & $-\frac{9}{20}$ & & \\
\hline 5 & $-\frac{1}{4}$ & $-\frac{13}{40}$ & & \\
\hline $2^{*}$ & $+\frac{3}{56}$ & $-\frac{1}{280}$ & $-\frac{3}{40}$ & {$[1,0]$} \\
\hline
\end{tabular}

TABLE II. The coefficients of shifts of the vibronic levels for second-order perturbation in the couplings $g_{2}$ and $g_{4}$, for the 0 - and 1-vibron multiplets of levels, for $n=1$ holes. In the first column we list the labels of the states according to spherical symmetry; the second and third column report the results of applying as a perturbation $H_{h-v}^{(2)}$ and $H_{h-v}^{(4)}$ alone respectively; in the next column the perturbation is done with equal couplings $g_{2}=g_{4}=g$. In this last case the true symmetry of the system is $\mathrm{SO}(5)$, that we evidence with the labels in the last column. The states marked with $\dagger$ and ${ }^{\ddagger}$, are multiply present in the 1-vibron multiplet, and therefore admix differently with the three different perturbations. Results in units of $\hbar \omega_{2}=\hbar \omega_{4}$. 


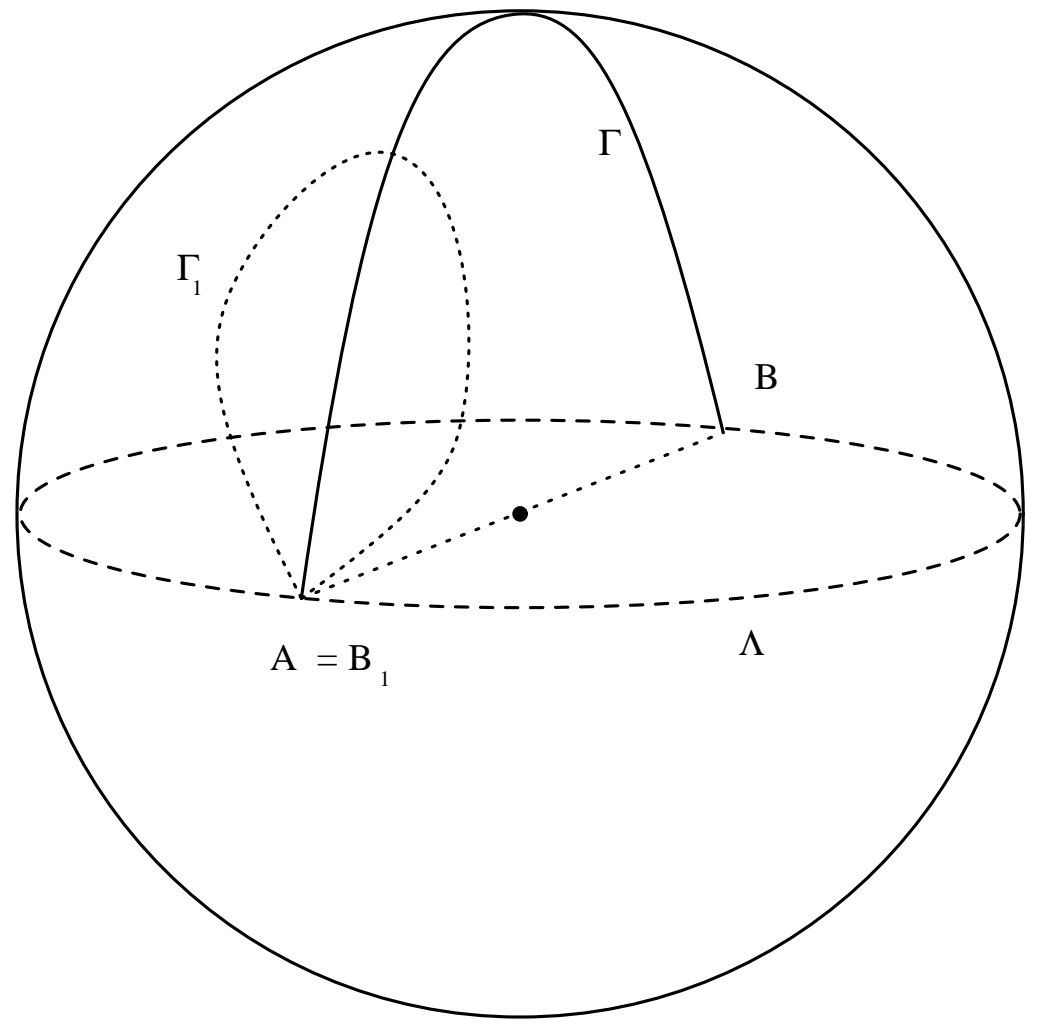

Fig. 1

De Los Rios, Manini, Tosatti 
Fig 2-De Los Rios, Manini, and Tosatti

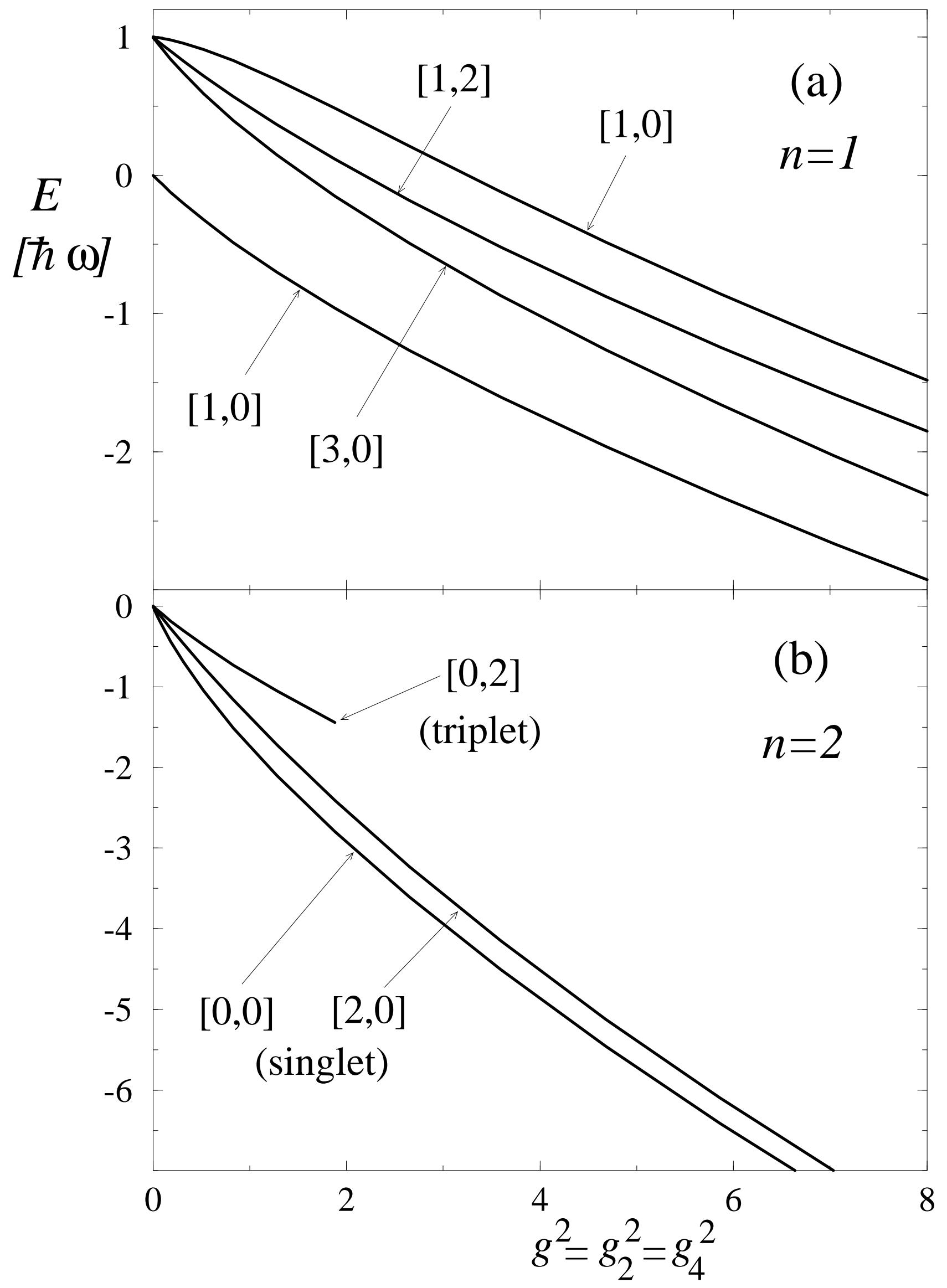


Fig 3 - De Los Rios, Manini, and Tosatti

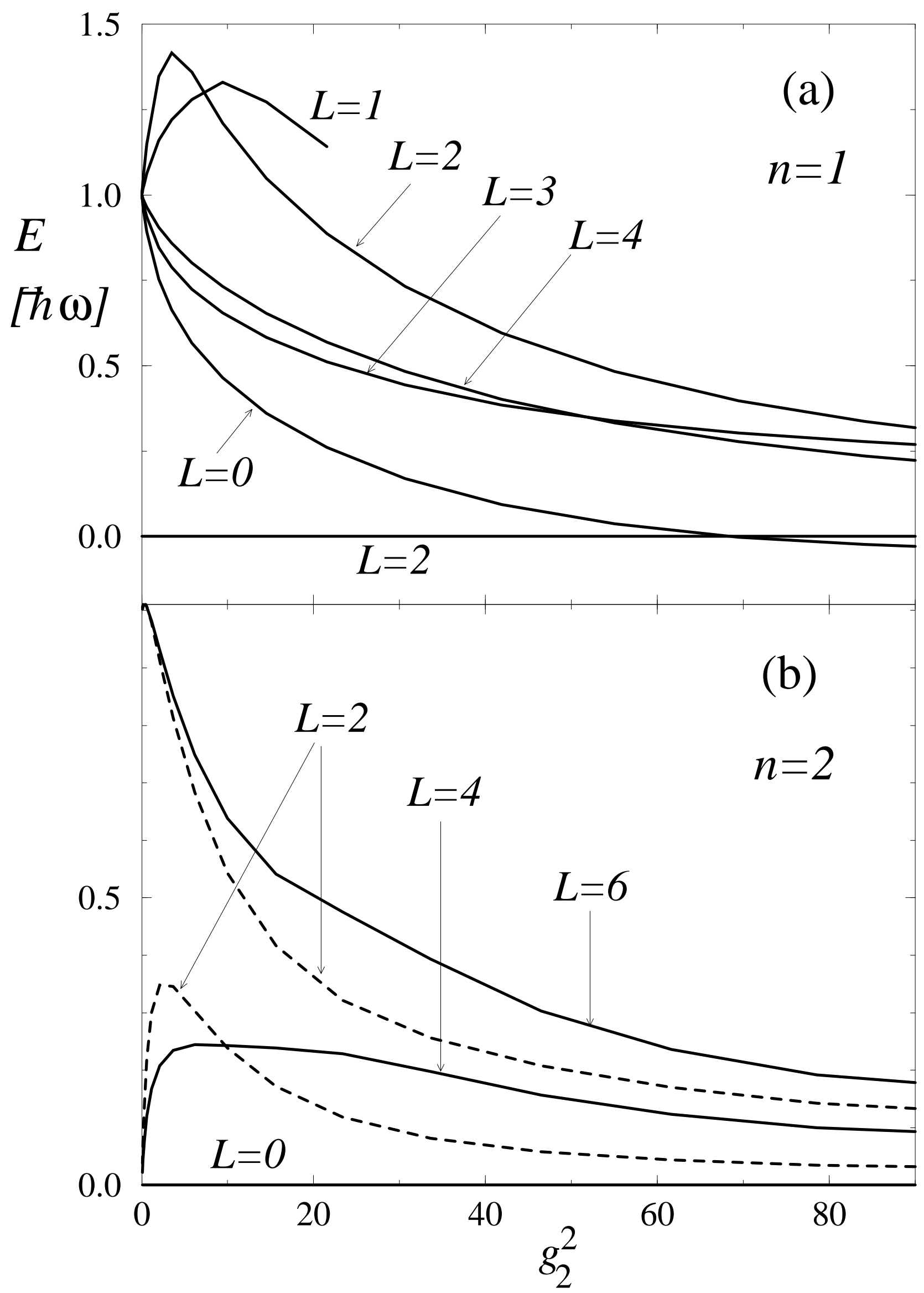

